\title{
LA INTEGRACIÓN DE LA EDUCACIÓN AMBIENTAL EN LA FORMACIÓN PERIODÍSTICA COMO PROYECTO DE INNOVACIÓN DOCENTE
}

\author{
María Teresa Mercado-Sáez¹. Universidad CEU Cardenal Herrera. España. \\ mmercado@uch.ceu.es
}

Sebastián Sánchez-Castillo. Universitat de València España.

Sebastian.sanchez@uv.es

\section{RESUMEN}

El reconocimiento del papel de los medios de comunicación como constructores de la realidad y como educadores informales en la sociedad actual inspira la acción educativa que se presenta como proyecto de innovación docente en la práctica universitaria. El objetivo de esta intervención pretende potenciar la reflexión crítica y activar el proceso enseñanza-aprendizaje en torno a la problemática ambiental relacionándola con la actualidad periodística y las rutinas productivas de los medios en la asignatura Fundamentos de la Comunicación Periodística que se imparte en el primer curso de los Grados de Ciencias de la Comunicación. En concreto, la integración de la Educación ambiental en la formación periodística se inicia con el análisis acerca de los conocimientos y creencias de los futuros comunicadores acerca del consumo energético o la conveniencia del uso de la energía nuclear. También se trata de comprobar los efectos sociales de los media en cuanto a la vinculación práctico-cognitiva tras el consumo (o no) de información periodística sobre la catástrofe de Fukushima. La finalidad última es por tanto la mejora de la calidad de la enseñanza universitaria para que el Periodismo contribuya a la protección medioambiental, tal y como se especifica en el RD 1393/2007.

PALABRAS CLAVE: Innovación docente - Fundamentos del Periodismo- Educación Ambiental

\footnotetext{
1 Autor correspondiente

María Teresa Mercado Sáez: Universidad CEU Cardenal Herrera. Valencia, España
} 


\title{
THE INTEGRATION OF ENVIRONMENTAL EDUCATION IN JOURNALISM STUDIESAS A TEACHING INNOVATION PROJECT
}

\begin{abstract}
The recognition of the role of the media as builders of reality and as informal educators in today's society inspires the educational activity that it is presented as a teaching innovation project in the university practice. The objective of this intervention aims at promoting critical reflection, and activating the teaching- learning process around environmental issues, which relates to current journalistic and production routines of the media in the subject Fundamentals of Communication Journalism that it is taught in the first year of Degrees of Communication Sciences. In particular, the integration of environmental education in journalism training begins with the analysis of knowledge and future communications beliefs about energy consumption, or the convenience o fusing nuclear energy. It also covers the social effects of the media in terms of practical-cognitive link after the consumption (or not) of the disaster in Fukushima media coverage. Therefore, the ultimate goal is to improve the quality of the Journalism university education and its contribution to environmental protection, as specified in RD 1393/2007.
\end{abstract}

KEY WORDS: Teaching innovation - Fundamentals of Journalism - Environmental Education

\section{INTRODUCCIÓN}

El proyecto de innovación educativa que se presenta responde al llamamiento de Naciones Unidas reclamando a los educadores de todos los niveles y áreas su contribución a formar ciudadanas y ciudadanos conscientes de la situación del mundo. En la "Década de la Educación por la Sostenibilidad" (2005-1015) ${ }^{2}$,la educación, tanto formal como informal, debe proporcionar una percepción correcta de los problemas y fomentar actitudes y comportamientos favorables para el logro de un desarrollo sostenible. Uno de estos problemas transnacionales son los ambientales. Al mismo tiempo, esta acción obedece a la necesidad de explorar nuevas fórmulas docentes mediante la experimentación de innovaciones en las aulas universitarias en el Espacio Europeo de Educación Superior. Como apuntaban Rekalde, Alonso, Arandia y Martínez:

\footnotetext{
${ }^{2}$ Proclamación de la Década de las Naciones Unidas de la Educación para el Desarrollo Sostenible. Resolución 57/254 aprobada por la Asamblea General de las Naciones Unidas el 20 de Diciembre de
} 
La reflexión sobre las formas de acción educativa en las aulas universitarias así como su consecuente innovación y mejora es una necesidad que sentimos como profesionales de la formación superior. Al mismo tiempo es una responsabilidad que asumimos en el marco de una institución que tiene como una de sus funciones principales contribuir y apoyar el avance del conocimiento (Rekalde et al, 2005, p. 65).

El objetivo fundamental de esta intervención pretende potenciar la reflexión crítica y activar el proceso enseñanza-aprendizaje en torno a la problemática ambiental relacionándola con la actualidad periodística y las rutinas productivas de los medios incidiendo en el primero de los objetivos de aprendizaje del Grado de Periodismo de la Universidad CEU Cardenal Herrera: desarrollar en los estudiantes el espíritu crítico y del conocimiento de su entorno necesarios para la búsqueda elaboración y gestión de la información ${ }^{3}$. En esta primera acción, nos centramos en el consumo energético y el debate en torno al uso de la energía nuclear como asunto central en la cuestión medioambiental.

De acuerdo con lo expuesto en el Libro Blanco y en el Programa Formativo de la titulación de Periodismo de la Universidad CEU Cardenal Herrera, el Grado de Periodismo tiene como objetivo general formar un/a profesional capacitado/a para buscar elaborar o gestionar información y contenidos para los medios de comunicación de la máxima calidad posible en cualquier situación, técnica, social o cultural.

'Fundamentos de la Comunicación Periodística', asignatura que se imparte en el primer curso de los Grados de Ciencias de la Comunicación de la Universidad CEU Cardenal Herrera (Periodismo, Publicidad y Relaciones Públicas, y Comunicación Audiovisual), es la materia más adecuada para esta acción educativa encaminada a integrar la problemática ambiental como primera toma de contacto del alumnado con el trabajo periodístico, es decir, con los procesos de selección, interpretación y narración de la actualidad así como en los modos de actuación profesional que se corresponden con ellos.

Entre las competencias genéricas (aplicaciones, aptitudes, destrezas y responsabilidades transversales) de la asignatura reflejadas en la guía docente figuran ser capaz de comprender y analizar proposiciones complejas con sentido crítico, en el contexto en el que se presentan; de reunir e interpretar datos relevantes para elaborar sus propias argumentaciones propias o de transmitir información, ideas, problemas y soluciones. Así, como afirma María Novo, titular de la Cátedra UNESCO de Educación Ambiental y Desarrollo Sostenible de la UNED:

\footnotetext{
${ }^{3}$ De acuerdo con lo expuesto en el Libro Blanco y en el Programa Formativo de la titulación de Periodismo de la Universidad CEU Cardenal Herrera, el Grado de Periodismo tiene como objetivo general formar un/a profesional capacitado/a para buscar elaborar o gestionar información y contenidos para los medios de comunicación de la máxima calidad posible en cualquier situación,
} 
La educación ambiental como tema transversal se orienta no solo a la comprensión y correcta interpretación de las cuestiones ambientales sino también a la necesidad de que los alumnos desarrollen valores acordes con tales planteamientos y elaboren propuestas alternativas orientadas a la toma de decisiones (Novo, 2005, p. 232).

Tomar conciencia de la trascendencia del Periodismo como constructor de la realidad social, por ejemplo en el sentido del que habla María Novo refiriéndose a las cuestiones medioambientales, es una de las competencias específicas que se adquieren en la materia según la memoria de grado verificada. El logro de esta competencia implica ser capaz de comprender que la labor del periodista -en los distintos ámbitos de la realidad en los que se despliega- tiene una particular trascendencia en la percepción de la realidad, la configuración de la ideología y la toma de decisiones de las personas, lo que comporta una considerable responsabilidad al tomar decisiones profesionales. La observación, análisis e interpretación de la realidad se traduce en un seguimiento sistemático de la actualidad como origen de la noticia.

Consideramos que la problemática ambiental en sus múltiples facetas debe formar parte de este seguimiento de la actualidad y estar integrada en las actividades globales dirigidas a los futuros comunicadores, aquellos que tendrán la posibilidad de actuar como educadores informales desde los medios, yendo más allá de los simples añadidos de temas ambientales a los contenidos.

La acción educativa se situaría entre la educación ambiental formal y no formal, ya que existe intencionalidad y especificidad, en la medida en que en el proyecto de acción comunicativa que se expone se desarrollan procesos que pretenden la modificación de conductas y en tanto que las actividades se realizan en instituciones educativas. Su intencionalidad obliga a desarrollar mecanismos de integración del tema ambiental en las actividades globales dentro de la asignatura 'Fundamentos de la Comunicación Periodística'.

La intervención incide en dos de las dimensiones de la calidad de la enseñanza definidas por Zabalza (Zabalza 2003, p. 210-214): la selección de contenidos y la metodología. En cuanto a la selección, se relacionan los contenidos o temas de la propia materia y/o de otras materias relacionadas y/o del mundo profesional combinando formación académica y la realidad de la crisis ambiental. 
En cuanto a la metodología, se utiliza un estilo retador y problematizante de aproximación a los contenidos tras el análisis de los conocimientos y creencias de los estudiantes sobre cuestiones energéticas. La finalidad última es por tanto la mejora de la calidad de la enseñanza universitaria para contribuir a los principios de protección medioambiental, tal y como se especifica en el RD1393/20074 .

\subsection{El Periodismo como educador informal}

La falta de concienciación ante la crisis ambiental se basa en creencias erróneas que se apoyan en la ausencia de información, en malas informaciones o en que aquellas que los receptores no están en condiciones de interpretar de forma crítica. Esto puede sortearse desde la comunicación, único instrumento que permite alcanzar la complicidad social (Castro, 2005).

Las campañas de instituciones públicas o privadas intentan motivar cambios en comportamientos por la sostenibilidad, pero son los medios de comunicación masiva los que con su labor informativa inciden en el nivel de interacción cognitivo entre persona-entorno y pueden convertirse en importantísimos instrumentos de sensibilización ambiental teniendo en cuenta que son la principal fuente de información de los ciudadanos ${ }^{5}$.

En este sentido, los medios de comunicación se constituyen en educadores informales considerando la educación ambiental informal como aquella que tiene lugar pese a la falta de intencionalidad educativa de quienes la promueven. Lo cual no significa, como en el caso de los medios de comunicación, que quienes escriben un artículo preparan un reportaje televisivo no deseen ayudar a las personas a formarse opiniones.

Lo que caracteriza a la educación informal es que ni el emisor ni el receptor se reconocen explícitamente como participantes de una acción comunicativa. No existen horarios ni reuniones entre quienes forman y quienes aprenden. Tampoco se dan las condiciones para una evaluación fiable de los objetivos conseguidos. Todo ello no merma las "posibilidades (y responsabilidades)" de quienes trabajan en el campo de la comunicación social a la hora de constituirse en educadores ambientales:

\footnotetext{
${ }^{4}$ REAL DECRETO 1393/2007, de 29 de octubre, por el que se establece la ordenación de las enseñanzas universitarias oficiales. BOE núm. 260, p. 44037. El párrafo en cuestión dice para contribuir al "conocimiento y desarrollo de los Derechos Humanos, los principios democráticos, los principios de igualdad entre mujeres y hombres, de solidaridad, de protección medioambiental, de accesibilidad universal y diseño para todos, y de fomento de la cultura de la paz".

5 Según el barómetro específico sobre el tema ambiental que elaboró el Centro de Investigaciones Sociológicas (CIS) en 2007, la mayor parte de los ciudadanos afirmaba haber tenido acceso a la información ambiental a través de los medios: un 87,9 por ciento en radio o TV; un 45,1 en prensa y un 1.5 .8 en Internet
} 
Muy al contrario, la conciencia de las enormes potencialidades no solo informativas, sino formativas, de los mensajes audiovisuales o escritos; la difusión masiva de noticias ambientales debe llevar a los periodistas a extremar su sensibilidad y cuidado a la hora de desarrollar su trabajo, de tal modo que sepan transmitir la gravedad de los problemas ambientales y presentar al mismo tiempo opciones y alternativas que ayuden a salir de la crisis (Novo, 2003, p. 225).

\subsection{Consumo energético y crisis ambiental}

Los temas que pueden tratarse dentro de la problemática ambiental son tantos ${ }^{6}$ que tras lo sucedido en Fukushima centramos la investigación-acción educativa en el debate en torno a la conveniencia o no del uso de la energía nuclear en el marco más amplio y complejo de la crisis energética vinculado al consumo.

El aumento del consumo es mucho más de lo que el planeta puede tolerar. En 2004, el Worldwatch Institute en su informe anual "El estado del mundo" contabilizaba 1.700 millones de personas como clase consumidora, es decir, aquella que ha adoptado el modo de vida occidental. Según el "Informe Planeta Vivo 2010" de WWF, una evaluación bianual que analiza la situación de la biodiversidad global y mide la demanda de la población sobre los recursos naturales de la Tierra, la huella ecológica se ha duplicado. Durante los años 70 la humanidad en su conjunto traspasó el punto en el que la Huella Ecológica y la biocapacidad anual de la Tierra estaban equiparadas.

Es decir, la población humana empezó a consumir recursos renovables más rápido de lo que pueden regenerar los ecosistemas y a liberar más CO2 de lo que los ecosistemas pueden absorber. Esta situación se denomina "translimitación ecológica" y ha continuado desde entonces. Actualmente, la demanda de la humanidad excede en cerca de un $30 \%$ la capacidad regeneradora del planeta, por lo que estamos destruyendo el capital natural en lugar de utilizar tan sólo los intereses, que sería lo sostenible. La organización insiste en que es imprescindible aunar los esfuerzos para cambiar el modelo energético actual (WWF 2010, p. 37).

\footnotetext{
${ }^{6}$ El periodista Joaquín Fernández (1995) elaboró una lista de los contenidos que ha de "afrontar el informador ambiental como aproximación orientativa": residuos, agua (ríos, pantanos, embalses, trasvases, regadíos, plantas desalinizadoras, confederaciones hidrográficas), bosques (incendios, limpieza, repoblaciones), protección de la naturaleza (espacios protegidos, parques naturales y nacionales, especies de fauna y flora en peligro de extinción, comercio y tráfico de animales, biodiversidad), mares (contaminación marina, disminución de recursos pesqueros, vertidos, mareas negras), costas (especulación urbanística, regeneración y protección de playas, banderas azules), suelo (especulación urbanística, erosión y desertificación), energía, transporte, atmósfera (contaminación atmosférica en general, capa de ozono, efecto invernadero, cambio climático), problemas urbanos (contaminación, tráfico, ruido, zonas verdes, degradación del patrimonio arquitectónico), agricultura), etcétera
} 
La World Energy Outlook (WEO -2009) de la Agencia Internacional de la Energía afirma que si los gobiernos mantienen sus programas y políticas actuales, la demanda mundial de energía se incrementará a un ritmo del 1,5\% anual durante el periodo 2007- 2030, desde los 12.000 millones de toneladas de petróleo equivalente hasta las 16.800. El rápido crecimiento económico y el consecuente aumento de la demanda energética que están experimentando numerosas naciones, principalmente en las del Sudeste asiático, incrementa la crisis ambiental.

Para el ex secretario de Estado de energía, Pedro Martín Uribe, "si todos los habitantes del planeta pudieran acceder a los mismos bienes y servicios a disposición de los ciudadanos de los países más desarrollados, el consumo energético mundial se multiplicaría por cuatro (...) El sistema energético actual impide que toda la población mundial pueda disfrutar de la misma calidad de vida, a la vez que sugieren que necesitamos un nuevo modelo energético que sea capaz de cubrir sus necesidades de una forma sostenible".

En esta reflexión, la catástrofe nuclear de Fukushima tras los terremotos y el posterior tsunami del 11 de marzo de 2011 ha intensificado el debate mundial acerca del papel de la energía nuclear en el futuro ante "la necesidad de impulsar un profundo cambio en nuestro paradigma energético para las próximas décadas, tanto desde el lado de la demanda, con medidas de ahorro y eficiencia energética, como desde el de la oferta, con fuentes de energía libres de carbono" (El País, "Alarma nuclear", 15 marzo 2011).

\section{METODOLOGÍA}

\subsection{Diseño de la investigación}

La planificación de la innovación docente en un contexto educativo específico constituye una primera fase del proyecto en la que se definen los objetivos expuestos y la metodología de la acción.

Para llevar a cabo este proyecto es necesario saber previamente qué piensan al respecto los estudiantes y se opta por ello por el diseño de un cuestionario como instrumento para obtener datos acerca de los conocimientos y creencias del alumnado acerca de la energía puesto que:

pedagógicamente, un planteamientos que no tenga en cuenta estas preconcepciones es un planteamiento incompleto. La construcción del conocimiento para ser efectiva debe apoyarse ineludiblemente en aquello que los sujetos piensan respecto del tema a desarrollar. (Novo, 2003, p. 172). 
Por ello, en una primera fase se analiza en profundidad qué opinan los futuros comunicadores sobre el consumo energético o la conveniencia del uso de la energía nuclear; qué saben y de dónde procede su información porque también se trata de comprobar los efectos sociales de los media como fuentes de información en cuanto a la vinculación práctico-cognitiva tras el consumo (o no) de información periodística sobre la catástrofe de Fukushima.

El diseño de investigación "integra, de modo coherente y adecuadamente correcto, técnicas de recogida de datos a utilizar, análisis previstos y objetivos" (Cea D’Ancona, 2001, p. 82). Los datos recogidos durante el proceso y su posterior análisis e interpretación, es decir, la evaluación de la innovación, nos permitirán orientar el diseño de recursos metodológicos para ofrecer diferentes vías de acceso a la temática ambiental y potenciar el aprendizaje significativo en torno a la problemática.

\subsection{Hipótesis}

Como hipótesis ${ }^{7}$ de partida, las cuales según Igartua (2006, p. 145) "presentan soluciones o respuestas provisionales a los problemas que ponen en marcha una investigación", se considera que aquellos estudiantes más informados mostrarán mayor preocupación por la crisis ambiental y que la catástrofe en Japón ha modificado sus opiniones al respecto del uso de la energía nuclear.

H1: Los estudiantes más informados mostrarán mayor preocupación por la crisis ambiental

H2: Tras la catástrofe de Fukushima, los estudiantes se muestran más contrarios a la energía nuclear.

Tras la creación de un libro de códigos con las variables consideradas se procedió al análisis correlacional multivariable junto a los estadísticos de contraste $x^{2}$ (Jicuadrado), y $r(r-y)$ de Pearson. Para evaluar la consistencia interna de la investigación se ha empleado el coeficiente $(a)$ de Cronbach.

Para profundizar más en el análisis del nivel de conocimientos y el nivel de actitudes y creencias de los estudiantes, y como complemento a la investigación central, consideramos oportuno comprobar si existen diferencias entre los estudiantes de Periodismo de primer curso de la asignatura de Fundamentos y los estudiantes de quinto curso de la optativa Periodismo Ambiental. Así, queda establecida una tercera hipótesis:

H3: Los estudiantes de Periodismo Ambiental de quinto curso están más informados y concienciados que los de primer curso de Fundamentos.

\footnotetext{
7 "Las hipótesis científicas son enunciados teóricos supuestos, no verificados pero probables, referentes a la relación entre variables" (Sierra Bravo, 1995, p. 74). Mediante las hipótesis "se permitirá la cuantificación de las variables, y presentados mediante la definición operativa" (Wimmer y Dominick, 1996, p.
} 


\subsection{Método}

El método de investigación utilizado es el correlacional utilizando como herramienta para la recogida de datos la encuesta ${ }^{8}$ mediante la que se recogen y analizan una serie de datos de una muestra de casos representativa de una población o universo más amplio, del que se pretende explorar, describir, predecir y/o explicar una serie de características.

Cada diseño de investigación requiere de un tipo de muestreo: "la elección entre la muestra probabilística y una no probabilística se determina con base en los objetivos del estudio, el esquema de la investigación y el alcance de sus contribuciones" (Sampieri, Fernández y Baptista, 1991, p. 209). Así, la muestra no probabilística de este estudio estuvo formada por 116 estudiantes de primer curso de Ciencias de la Información: 51 de Periodismo (44\%), 40 de Publicidad y Relaciones Públicas (34,5\%) y 25 de Comunicación Audiovisual $(21,6 \%)$. Con una edad comprendida entre los 1832 años, las mujeres representan el 66,4\% de los alumnos que se encontraban en clase de la asignatura Fundamentos de la Comunicación Periodística los días 12 y 16 de mayo de 2011.

Para la verificación de la tercera hipótesis se seleccionan 28 estudiantes de Periodismo para igualar el número de alumnos y alumnas matriculados en la optativa de quinto curso, lo que supone una muestra representativa aceptable y con una consistencia interna confirmada. A partir de ese universo se establecieron niveles de correlación y la articulación de tablas de contingencia explicativas.

La recogida de los datos se realizó colectivamente en la Universidad CEU Cardenal Herrera de Valencia en el contexto del grupo-aula, bajo condiciones de disponibilidad y participación voluntaria. Los estudiantes cumplimentaron un cuestionario ad hoc. Algunas de los enunciados de la encuesta en relación a la cuestión energética fueron si consideraban que la escasez de energía puede ser uno de los problemas más importantes que tendrán que afrontar los países occidentales o si estaban de acuerdo o en desacuerdo con afirmaciones sobre las energías renovables y la nuclear ${ }^{9}$.

Otras cuestiones pretendían evaluar el grado de conocimiento sobre la relación entre

\footnotetext{
${ }^{8}$ La encuesta se puede utilizar no sólo para realizar investigaciones de tipo exploratorio o descriptivo, sino también para analizar y explicar las relaciones existentes entre diferentes y múltiples variables y fenómenos sociales (Marsh, 1982; De Vaus, 1993).

9 - La energía que proporcionan el petróleo, el carbón y el gas, podrá ser sustituida a largo plazo (Alt1).

- Las energías alternativas (solar, eólica, biomasa) son eficaces (Alt2).

- Las energías alternativas (solar, eólica, biomasa) no pueden suministrar toda la energía que se necesita en un país (Alt3).

- La energía nuclear es la energía es segura, competitiva y garantiza el suministro eléctrico (Nucl1).

- La energía nuclear debe desaparecer porque genera residuos altamente radiactivos, muy peligrosos para la salud y el medio
} 
tipo de energía y contaminación. Por ejemplo, se les preguntaba cuál era la energía que se debería utilizar menos ofreciéndoles un listado (petróleo, gas, carbón, nuclear, sol, eólica, hidráulica, biomasa). Para comprobar si realmente siguen la información que ofrecen los medios, se opta por presentar una pregunta control: ¿Cuántos años hace de la catástrofe de Chernóbil? Todos los medios informativos han ofrecido reportajes sobre lo qué pasó y los efectos en la zona hoy en día cuando se cumplen 25 años de la catástrofe nuclear ${ }^{10}$.

El aniversario podría haber pasado más o menos desapercibido, pero el temor surgido por la situación de la central de Fukushima en Japón, ha hecho que se ponga más atención sobre las consecuencias de los accidentes nucleares. Interesa conocer si se sienten bien informados sobre lo sucedido en Japón y si han modificado su forma de pensar al respecto del uso de la energía nuclear.

\subsection{Estadísticos}

Para la resolución cuantitativa de la investigación se emplearon varios estadísticos con los que resolver las hipótesis de partida y conocer el grado o fuerza de asociación entre las variables propuestas. Bajo los presupuestos de la estadística inferencial, y mediante el proceso de contraste de hipótesis, se intentará resolver el planteamiento inicial de la investigación. Los objetos de investigación, es decir, las respuestas a las encuestas serán analizadas y sometidas al rigor del análisis univariable, resolviendo así tanto las hipótesis de investigación como la hipótesis estadística.

Para el proceso analítico y de asociaciones se usó el análisis de contingencia ya que este proceso "se dedica a inferir la red de asociaciones de una fuente a partir de la pauta de co-ocurrencia de símbolos en los mensajes (...) el análisis de contingencia es una técnica analítica por derecho propio" (Krippendorff, 1990: 168-169).

De esta manera, para el análisis completo de las inferencias, y empleando el software estadístico SPSS v.17.0, se procedió a la realización de una tabla de contingencia o Crosstabulation, ya que se trata de uno de los procedimientos más utilizados en la investigación de análisis de contenido en comunicación, por la posibilidad de aplicar índices de fiabilidad intercodificadores, como el coeficiente Kappa de Cohen o la Pi de Scott (Neuendorf, 2002); (Igartua, 2006); (Wimmer \& Dominick, 1996); (D’Ancona, 2001).

En la resolución de las correlaciones se ha empleado la prueba de $\left(\mathrm{X}^{2}\right)$ Chi-cuadrado (Chi-Square Tests). Este estadístico $X^{2}$ se obtiene de la comparación entre las

\footnotetext{
${ }^{10}$ En las primeras horas de la mañana del 26 de abril de 1986 explotó la unidad 4 del reactor nuclear de Chernóbil, en Ucrania, liberando una radioactividad decenas de veces mayor que la producida por la bomba atómica lanzada sobre Hiroshima en 1945. Dejó como legado una ciudad abandonada, 31 muertos, efectos devastadores para la salud de millones de personas y la desconfianza del mundo en la eneróía nisclear
} 
frecuencias observadas y aquellas que cabría esperar en caso de inexistencia de relación entre las variables (D’Ancona, 2001:335). Se acepta para este estadístico un nivel de probabilidad $(p)$ de 0,05 (Wimmer \&Dominick, 1996).

Por otra parte, para establecer el grado o fuerza de asociación entre las variables más importantes para la resolución de las hipótesis, se empleó el estadístico $r$ de Pearson $(r-y)$ con el que determinar el grado de covariación conjunta entre las variables expuestas de forma lineal, al menos con una escala de intervalo y siempre teniendo en cuenta el signo del coeficiente. Es necesario recordar que el signo de un coeficiente de correlación indica la forma en que dos variables están linealmente asociadas.

Es positivo cuando la asociación entre dos variables es directa, es decir, cuando a medida que crece el valor de $X$ crece también el valor de Y. Será negativo cuando es inversa, y a medida que crece el valor de $X$ decrece el valor de $Y$. Por otra parte, un valor de 0 indica que no existe asociación lineal, aunque esta puede ser curvilínea. Por ello no debe interpretarse como la ausencia de asociación, sino como ausencia de asociación lineal (Igartua, 2006, p. 516).

\section{ANÁLISIS Y DISCUSIÓN}

En primer lugar, el cuestionario pregunta cuáles son los principales problemas del país y cuáles les afectan más personalmente. Los estudiantes han de elegir de una lista cerrada elaborada con las respuestas más habituales recogidas en los barómetros del CIS11. En su mayoría se muestran preocupados por el paro (72\%) y la crisis económica pero es la educación el problema que más les afecta $(26,7 \%)$.

De 30 respuestas, hay 4 relacionados con el medio ambiente que han sido mencionadas en 30 ocasiones $(0,15 \%)$, ninguna de ellas como principal problema. Es significativo que la pérdida de biodiversidad solo aparece una vez mientras que el cambio climático y la dependencia energética lo hacen en 14 y 12 respectivamente. Por otra parte 5 alumnos han señalado los problemas medioambientales en general.

Al ser preguntados directamente sobre si la escasez energética puede ser un problema, el 82,8 por ciento responde afirmativamente $(n=96)$. Sin embargo, este porcentaje se reduce a la hora de valorar si consumir menos mejoraría nuestro nivel de vida ( $n=65$; un 56\%). La inmensa mayoría de los alumnos $(92,2 \%)$ afirma que les interesa el medio ambiente y el 89,7\% opina que esté en peligro aunque al mismo

\footnotetext{
${ }^{11}$ El paro, las drogas, la inseguridad ciudadana, el terrorismo, las infraestructuras, la sanidad, la vivienda, los problemas de índole económica, el cambio climático, la corrupción y el fraude, la dependencia energética del petróleo, las guerras en general, la Administración de Justicia, el racismo, la inmigración, la violencia contra la mujer, los problemas relacionados con la juventud, la crisis de valores, la educación, los problemas medioambientales en general, el Gobierno, los políticos y los partidos, el funcionamiento de los servicios públicos, los nacionalismos, los problemas relacionados con la mujer, el terrorismo internacional, la pérdida de biodiversidad, las preocupaciones y situaciones nersonales. Otras resnisestas. Ninowino v N.S
} 
tiempo, el 20,7\% cree que tiene cosas más importantes en las que pensar que el medio ambiente.

Más divididos aparecen en relación a los comportamientos puesto que el 33,6\% piensa que lo que hace como individuo no tiene impacto en el entorno si el resto de ciudadanos no hacen lo mismo y sobre todo, el 50,9 por ciento está muy de acuerdo con que su acción individual no tiene impacto si las empresas o industrias contaminantes no hacen más esfuerzos por proteger el medio ambiente.

Sólo 4 personas $(3,4 \%)$ afirman no haber recibido información sobre el proceso de cambio climático ni sobre la forma de reducir el consumo de energía. Ninguna de las 4 responden a las cuestiones con las que podemos comprobar el nivel de información o seguimiento de la actualidad, sobre todo la que se refiere al $25^{\circ}$ aniversario de Chernóbil (se les pregunta cuántos años hace de la catástrofe). Esta cuestión ha sido respondida de manera correcta por el $41,4 \%$ de los alumnos $(n=48)$, ver tabla 1.

Tabla 1. Encuesta. Elaboración propia

\begin{tabular}{lcc}
\hline \multicolumn{1}{c}{ Chernóbil } & Frecuencia & Porcentaje \\
\hline Correcto & 48 & $41,4 \%$ \\
No correcto & 20 & $17,2 \%$ \\
N/S & 48 & $41,4 \%$ \\
Total & $\mathbf{1 1 6}$ & $\mathbf{1 0 0 , 0} \%$ \\
\hline
\end{tabular}

De este tipo de alumno mejor informado que el resto, han contestado "muy de acuerdo" en a 'Nuestra calidad de vida mejoraría se gastáramos menos electricidad, gas o gasolina' el 56\% ( $n=27)$. Tan sólo $(n=8,16 \%)$ han contestado "muy en desacuerdo". Los alumnos indecisos suponen el (n=11, 23\%).

El 24,13\% de los estudiantes estarían dispuestos a cambiar sus hábitos de consumo y/o estilo de vida 'Con toda seguridad' $(n=28)$ y el 42, 24\% 'Probablemente' ( $n=49)$. Sólo 8 personas responden 'Probablemente no' (6,89\%) y dos no lo saben $(1,72 \%)$. Ninguna contesta 'No con toda seguridad'.

Más de la mitad de los alumnos encuestados $(n=62)$ aseguran que tras lo sucedido en Japón ha cambiado su forma de pensar al respecto del uso de la energía nuclear" (un $53,4 \%)$. De estos estudiantes, el 72,5\% ( $n=45)$ ha señalado que "la energía nuclear debe desaparecer porque genera residuos altamente radiactivos, muy peligrosos para la salud y el medio ambiente". Al mismo tiempo, la mayoría de los que afirman que no ha cambiado su forma de pensar $(n=31)$ se muestran también claramente en contra $(77,5 \%)$. 
Del grupo que dice no estar suficientemente informado de lo sucedido en Japón, $\mathrm{n}=39 ; 33,6 \%$, el 9,4\%; $\mathrm{n}=11$ no van a cambiar su forma de pensar (menos concienciados). Sin embargo, $\mathrm{n}=16 ; 13,79 \%$ están indecisos. En cuanto a las correlaciones de dependencia, no existe una relación significativa entre el sexo del encuestado y los hábitos de consumo y/o estilo de vida, [( $\left.X^{2} 4, N=116\right)=2,413$, $\mathrm{p}<, 660]$. Sin embargo, las mujeres, con un $23,27 \%$ han contestado que si cambiarían de hábitos con toda probabilidad para adaptarse al cambio climático. Sólo el $10,34 \%$ de hombres.

Consideramos concienciados a los estudiantes que han respondido 'muy de acuerdo' a las frases 'La energía que proporciona el petróleo, el carbón y el gas, podrá ser sustituida a largo plazo' (Alt1), 'Las energías alternativas (solar, eólica, biomasa) son eficaces' (Alt 2), 'La energía nuclear debe desaparecer porque genera residuos altamente radiactivos, muy peligrosos para la salud y el medio ambiente' (Nuc2) y 'Nuestra calidad de vida mejoraría si gastáramos menos electricidad, gas o gasolina' (Cal) y muy en desacuerdo a 'Las energías alternativas (solar, eólica, biomasa) no pueden suministrar toda la energía que se necesita en un país' (Alt3) y 'La energía nuclear es la energía es segura, competitiva, limpia y garantiza el suministro eléctrico' (Nuc1) y 'Consumir menos electricidad, gas o gasolina reduciría nuestro nivel de vida' (Con2).

Los informados son aquellos que responden correctamente a la cuestión acerca de cuáles son las actividades que emplean más energía (Act 1 y 2 ), afirman haber recibido información acerca del cambio climático (CC) sobre el debate nuclear (Mnuclear), sobre la forma de reducir el consumo de energía (Inforeducir1) y sobre lo sucedido en Japón (Japón1). Además, saben cuándo sucedió el accidente de Chernóbil. Como "muy informados", tan sólo a 6 personas; el 5,17\% de los jóvenes encuestados han contestado positivamente a las preguntas "se considera

suficientemente informado de la noticia de Japón", "cuantos años hace de Chernóbil", y "cuantas centrales hay en España".

La fuerza en la correlación entre el grupo de alumnos informados y concienciados, en la tabla 2 se indica el grado de asociación entre ambos. 
Tabla 2. El grado de asociación entre alumnos informados y concienciados

\begin{tabular}{|c|c|c|c|c|c|c|}
\hline \multicolumn{7}{|c|}{ Correlación Pearson $(x-y)^{12}$} \\
\hline \multicolumn{2}{|c|}{ Informados } & \multicolumn{2}{|c|}{ Concienciados } & \multirow[b]{2}{*}{ Cal } & \multirow[b]{2}{*}{ Nuc1 } & \multirow[b]{2}{*}{ Nuc2 } \\
\hline & Alt1 & Alt2 & Alt3 & & & \\
\hline Ac1 & ,022 &,- 073 & 142 &,- 073 &, 064 &,- 067 \\
\hline Ac2 &,$- 221\left(^{*}\right)$ &,- 111 &,- 120 & 033 &,- 046 & 100 \\
\hline 2010 &,- 077 &,- 040 &,- 114 & 061 & ,064 &,- 034 \\
\hline Con1 &,- 045 & ,065 & 146 & ,049 & ,009 & ,024 \\
\hline Con2 &,- 082 &,- 060 & $209\left(^{*}\right)$ & 138 & ,098 &,- 082 \\
\hline MN & 052 &,- 085 &,- 096 & 088 &,- 035 & ,068 \\
\hline IR1 & 123 &,- 054 &,- 029 &,- 009 & ,067 & ,010 \\
\hline IR2 & 079 &,- 082 & 146 &,- 093 & 149 & ,068 \\
\hline J1 & $\left.262^{(* *}\right)$ & $\left.187^{*}\right)$ &,- 060 &,- 054 &,- 050 & ,058 \\
\hline $\mathrm{J} 2$ & ,094 & ,093 &,$- 207\left(^{*}\right)$ &,- 002 & ,004 & 114 \\
\hline
\end{tabular}

*. La correlación es significante al nivel 0,05 (bilateral).

**. La correlación es significativa al nivel 0,01 (bilateral).

En la resolución de la hipótesis $\mathrm{n}^{\circ} 3$, ha quedado de manifiesto que el grado de concienciación y de información medioambiental de los alumnos es claramente dependiente del curso académico al que asisten en algunas cuestiones. De esta forma, las variables 'Fuentes' (De las fuentes de energía utilizadas en nuestro país, ¿cuál cree que se debería utilizar menos en primer lugar?), 'Calidad' (Nuestra calidad de vida mejoraría si gastáramos menos electricidad, gas o gasolina ), 'Agua' (Es imposible reducir el consumo de agua en las sociedad avanzadas), 'Gases' (El incremento de gases de efecto invernadero es un proceso natural), 'Catástrofe' (El calentamiento global va a provocar, entre otras cosas, el deshielo de los glaciares y el consiguiente aumento del nivel del mar), 'Medios Nuclear' (¿Ha visto, oído o leído en algún medio de comunicación algo sobre la conveniencia o no del uso de la energía nuclear?) han resultado significativas.

\footnotetext{
${ }^{12}$ Informados: Ac1 (Actividad 1);Ac2 (Actividad 2);2010 (CC2010);Con1 (Consumo 1);Con2 (Consumo 2);MN (Medios y E.Nuclear);IR1 (Info reducir 1);IR2 (Info Reducir 2);J1 (Info Japón 1);J2 (Info Japón 2). Concienciados: Alt1 (Alternativa 1); Alt2 (Alternativa 2);Alt3 (Alternativa 3);Cal (Calidad);Nuc1 (Nucleares 1);Nuc2 (Nucleares 2).
} 
En la tabla 3, la resolución de la tabla de contingencia con dichas variables ha resultado ser muy poco homogénea. La energía nuclear es la que goza de menor aceptación entre los alumnos y la hidráulica la que es considerada como más sostenible, además su correlación es muy significativa $\left[X^{2}(6, N=56)=23,386, p<\right.$ $, 001]^{13}$, y valor $\operatorname{Eta}^{14} \eta=$,646. Sin embargo, ningún estudiante de primero señala el carbón como fuente contaminante mientras que los de quinto la señalan en un 42,9\%, un porcentaje mayor incluso que el de los que opinan que se debería utilizar menos petróleo.

Los de primer curso creen que la energía a desterrar es la nuclear, precisamente después del accidente de Fukushima. Solo un 14,3 por ciento de los estudiantes de quinto, con un mayor conocimiento acerca de las fuentes de energía y del consumo, piensan que es la nuclear la que debe dejar de utilizarse en relación a la contaminación.

Tabla 3. Crosstabs

Fuentes. Elaboración propia

\begin{tabular}{|c|c|c|c|c|c|c|c|}
\hline \multicolumn{8}{|c|}{$\begin{array}{l}\text { Fuentes. De las ocho fuentes de energía utilizadas en nuestro país, ¿cuál cree que se } \\
\text { debería utilizar menos en primer lugar? }\end{array}$} \\
\hline & Carbón & Nuclear & Petróleo & Hidráulica & Gas & Ninguna & ns/nc \\
\hline Curso $1^{\circ}$ & 0 & $\begin{array}{c}15 \\
53,6 \% \\
\end{array}$ & $\begin{array}{c}10 \\
35,7 \% \\
\end{array}$ & $\begin{array}{c}1 \\
3,6 \% \\
\end{array}$ & 0 & $\begin{array}{c}1 \\
3,6 \% \\
\end{array}$ & $\begin{array}{c}1 \\
3,6 \% \\
\end{array}$ \\
\hline Curso $5^{\circ}$ & $\begin{array}{c}12, \\
42,9 \%\end{array}$ & $\begin{array}{c}4 \\
14,3 \%\end{array}$ & $\begin{array}{c}10 \\
35,7 \%\end{array}$ & 0 & $\begin{array}{c}2 \\
7,1 \%\end{array}$ & 0 & 0 \\
\hline
\end{tabular}

Respecto a las variables dicotómicas (sin tener en cuenta el ítem ns/nc), los alumnos respondieron a las cuestiones con las afirmaciones "muy de acuerdo" o "muy en desacuerdo", a temas sobre "Calidad" [X2(2, N=56)=7,932, $\mathrm{p}<, 019], \mathrm{\eta}=$,376, en la variable "Agua 2" [X²(2, N=56)=6,954, $\mathrm{p}<, 031], \eta=, 352$, en “Gases 2 " [X2 $(2, N=56)=$ $7,500, \mathrm{p}<, 024], \mathrm{\eta}=$,366, y finalmente "Catástrofe", $\left[X^{2}(2, N=56)=6,190, \mathrm{p}<, 045\right], \eta=$ ,332, ver tabla 4 .

\footnotetext{
${ }^{13}$ Para la descripción de las pruebas de contraste de hipótesis se aceptan las normas APA (American Psychological Association)

${ }^{14}$ Eta ( $\left.\eta\right)$, es una medida de asociación cuyo valor siempre está comprendido entre 0 y 1 . El valor 0 indica que no hay asociación entre la variable de fila (la cuantitativa) y de columna (la cualitativa
} 
Tabla 4. Crosstabs (Elaboración propia)

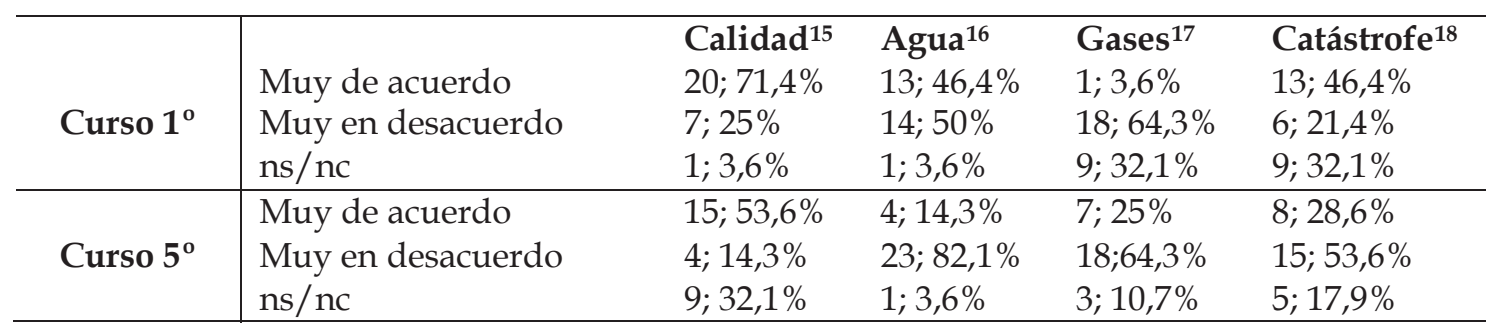

Respecto a las variables no dicotómica 'Medios Nuclear' difieren según el curso. Podemos observar como los estudiantes de quinto consumen más información en medios escritos que los de primero.

El dato más significativo es el alto porcentaje de alumnos de primero de Periodismo que reconocen no haber recibido ninguna información acerca de la conveniencia o no del uso de la energía nuclear de los medios: un $25 \%$. Según se desprende de la tabla 5 , todos los alumnos de quinto sí han recibido esa información.

Tabla 5. Crosstabs (Elaboración propia)

\begin{tabular}{l} 
Medios Nuclear: ¿Ha visto, oído o leído en algún medio de comunicación algo sobre la \\
conveniencia o no del uso de la energía nuclear?) \\
\hline
\end{tabular}

\footnotetext{
${ }^{15}$ Afirmación: "Nuestra calidad de vida mejoraría si gastáramos menos electricidad, gas o gasolina"

${ }^{16}$ Afirmación: "Es imposible reducir el consumo de agua en las sociedad avanzadas (nutrición, higiene, agricultura)"

${ }^{17}$ Afirmación: "El incremento de gases de efecto invernadero es un proceso natural"

${ }^{18}$ Afirmación: “El proceso de calentamiento global es irreversible y sus consecuencias para el planeta catastróficas"
} 


\section{CONCLUSIONES}

La capacidad de comprensión y análisis de proposiciones complejas como el paradigma energético mundial puede incrementarse fundamentando y poniendo en marcha en el aula métodos de trabajo que potencien el aprendizaje significativo de los estudiantes de Comunicación en torno a la problemática ambiental. La integración de la Educación ambiental en la formación periodística no supondría la teorización sobre ecología y medio ambiente sino la reflexión crítica acerca de cómo informar sobre problemas como la escasez energética, es decir, la incorporación de esta temática en los procesos de selección, interpretación y narración de la actualidad así como en los modos de actuación profesional que se corresponden con ellos.

Consideramos que en el contexto de una colaboración multidisciplinar, las ciencias de la Comunicación unidas a la psicología ambiental pueden desarrollar significativas aportaciones para diseñar, implementar y evaluar estrategias sociales y educativas más eficientes y efectivas a favor de las sostenibilidad, tal como ha indicado Ricardo de Castro (Castro, 2004).

Los resultados de la investigación llevada a cabo muestran que la mayoría de los alumnos muestran interés por los problemas ambientales aunque no tanto por el cambio de conductas individuales para protegerlo. Confirmando la hipótesis inicial, los alumnos más informados sobre cuestiones relativas a energía y medioambiente muestran una mayor sensibilidad y por lo tanto mayor preocupación por la crisis ambiental.

La catástrofe de Fukushima, tal y como la han contado los medios de comunicación, ha modificado la percepción acerca de la energía nuclear de más de la mitad de los estudiantes encuestados situándose en contra. Los alumnos que manifiestan no haber cambiado de opinión están también claramente en contra, es decir, no ha habidocambio puesto que ya opinaban así. La información sobre la catástrofe ha reafirmado sus ideas.

Nada extraño puesto que este suceso ha impactado con fuerza en la opinión pública de todo el mundo. En España no lo ha hecho tanto como en los países asiáticos que lo han vivido de cerca (Corea del Sur, China, India y en el propio Japón), pero el 18\% de las personas que se oponen hoy a la energía nuclear se han puesto en contra de ella tras el accidente. Así lo asegura una encuesta hecha por la consultora Ipsos en 24 países (El País, 25 de junio 2011). De media en todos ellos, un 26\% de opositores a las nucleares lo son por Fukushima. En España, las cifras globales -un $60 \%$ de la población está en contra de la energía nuclear- concuerdan con las que el Centro de Investigaciones Sociológicas publicó a primeros de junio. 
En definitiva, el desarrollo de mecanismos de integración de temas ambientales en las actividades globales dentro de la asignatura 'Fundamentos de la Comunicación Periodística' favorece la sensibilización de los estudiantes respecto a la protección medioambiental.

\section{REFERENCIAS}

Bisquerra Alzina, R. (2009). Metodología de la investigación educativa. Madrid: La Muralla.

Castro, R. de (2004). Persona, cultura y medio ambiente. ¿Qué puede aportar la psicología ambiental a la educación para la sostenibilidad? En Ciclos. Cuadernos de Comunicación, interpretación y educación ambiental, 15: 3-7.

Castro, R. de (2005). Más que palabras. Comunicación ambiental para una sociedad sostenible. Valladolid: Junta de Castilla y León. Gea.

Cea D’ancona, M.A (2001). Metodología cuantitativa. Estrategias y técnicas de investigación social. Madrid: Síntesis Sociología.

Duarte, Carlos (2006). Cambio Global. Impacto de la actividad humana sobre el sistema Tierra. Madrid: CSIC.

Fernández Sánchez, Joaquín (1995). Periodismo ambiental en España. Madrid: Ministerio de Obras Públicas, Transportes y Medio Ambiente.

García-Valcárcel Muñoz-Repiso, A. (2009). Experiencias de innovación docente universitaria. Salamanca: Universidad de Salamanca.

Gil Pascual, J. A. (2004). Bases metodológicas de la investigación educativa: análisis de datos. Madrid: UNED.

Goñi Grandmontagne, A. (2005). Innovación educativa en la Universidad. Bilbao: Universidad del País Vasco.

Igartua, J. J. (2006). Métodos cuantitativos de investigación en comunicación. Barcelona: Bosch.

Latorre Beltrán, A. et al. (2003). Bases metodológicas de la investigación educativa. Barcelona: Experiencia.

López Pintor R. (1992). El análisis de los datos de encuesta. En GARCÍA El análisis de la realidad social. Madrid: Alianza Editorial. 
Martín Uribe, P. L. (2011). Energías renovables: ¿estamos preparados?. en El País.

Meira, P. A. (2006). Crisis ambiental y globalización: una lectura para educadores ambientales en un mundo insostenible. Trayectorias, 120(21): 110-122.

Novo, MARÍA (2003): La educación ambiental. Bases éticas, conceptuales y metodológicas. Editorial Universitas. Madrid.

Rekalde, I., Alonso, J., Arandia, M.; Martínez, I. (2005). La metodología dialógica al servicio de una universidad de calidad: Articulando distintas herramientas didácticas. En Innovación educativa en la Universidad (pp. 65-77). Bilbao: Universidad del País Vasco.

Rojas Tejada, A. J. et al. (1998). Investigar mediante encuestas. Fundamentos teóricos y aspectos prácticos. Madrid: Síntesis.

Vilches, Amparo y Gil Pérez, Daniel (2007): “Emergencia planetaria: necesidad de un planteamiento global" en Educatio siglo XXI, Vol 25. Universidad de Murcia. Murcia. Pág. 19-49

Wimmer, R. D. \& Dominick, J. R (1996). La investigación científica de los medios de comunicación. Una introducción a sus métodos. Barcelona: Bosch.

Zabalza, M. A. (2003). Competencias docentes del profesorado universitario. Madrid: Narcea.

\section{María Teresa Mercado Sáez}

Doctora en Ciencias de la Información (UCM, 2005), licenciada en Periodismo (CEU San Pablo-UPV, 1996) y en Humanidades (UV, 2007), Máster en Gestión Cultural (UCH, 2005) y Especialista Universitario en Educación Ambiental (UNED, 2010).Profesora en el Departamento de Comunicación y Periodismo de la Universidad CEU UCH en Valencia, imparte las asignaturas de Fundamentos de la Comunicación Periodística y Periodismo Ambiental. Subdirectora del Instituto de Investigación CEU de Estudios y Disciplinas Ambientales y miembro del Grupo de Investigación en Discapacidad y Comunicación (GIDYC) en la CEU UCH, de la Asociación Española de Investigación en Comunicación AE-IC y de la Asociación de Periodistas Ambientales. Ha publicado artículos sobre periodismo ambiental, el tratamiento informativo de la discapacidad y el patrimonio cultural como especialización periodística. 


\section{Sebastián Sánchez Castillo}

Doctor en Ciencias de la Información (UV, 2008), licenciado en Historia (UV, 2003) y en Comunicación Audiovisual (UOC, 2004). Profesor del Departamento de Teoría de los Lenguajes y Ciencias de la Comunicación de la Facultad de Filología, Traducción y Comunicación de la Universidad de Valencia. Su perfil docente se centra en la Realización Televisiva, Diseño de Proyectos Interactivos y Tecnologías de la Comunicación, asignaturas del Grado de Comunicación Audiovisual y del Máster de Contenidos y Formatos Audiovisuales en la era Digital. Miembro del Grupo de Investigación en Discapacidad y Comunicación (GIDYC) en la CEU-UCH y de la Asociación Española de Investigación en Comunicación AE-IC (estudios de audiencia y recepción). Al respecto, ha publicado diversos artículos empleando metodologías cuantitativas bajo un enfoque empírico-conceptual y de análisis estadísticos de naturaleza inferencial y multivariable. 


\section{ANEXO}

\section{Resultados encuesta estudiantes primer curso Fundamentos de la Comunicación Periodística.}

En su opinión, ¿la escasez de energía puede ser uno de los problemas más importantes que tendrán que afrontar los países occidentales?

Sí: $(\mathrm{n}=96 ; 82,8 \%)$

No: $(n=9 ; 7,8 \%)$

NS/NC: $(n=11 ; 9,5 \%)$

De las ocho fuentes de energía utilizadas en nuestro país ¿cuál cree que se debería utilizar menos en primer lugar?

$1^{\circ}$. Nuclear $(\mathrm{n}=61 ; 52,6 \%)$

$2^{\circ}$. Petróleo $(n=38 ; 32,8 \%)$

¿Ha visto, oído o leído en algún medio de comunicación algo sobre el proceso de calentamiento del planeta y del cambio climático?
a) Sí, en prensa $(n=6 ; 5,2 \%)$
b) Sí, en tv ( $\mathrm{n}=17 ; 14,7 \%)$
c) Sí, en Internet $(\mathrm{n}=1 ; 0,9 \%)$
d) Sí, en radio $(0 \%)$

Sí, en varios medios, incluida la tv $(\mathrm{n}=87 ; 75 \%)$

a) No, en ningún medio $(n=4 ; 3,4 \%)$

b) ¿Está más bien de acuerdo o más bien en desacuerdo con estas frases?

c) La energía que proporciona el petróleo, el carbón y el gas, podrá ser sustituida a largo plazo (Alt1)

Muy de acuerdo $(\mathrm{n}=88 ; 75,9 \%)$

Muy en desacuerdo $(\mathrm{n}=15 ; 12,9 \%)$

NS/NC ( $n=13 ; 11,2 \%)$

a) Las energías alternativas (solar, eólica, biomasa) son eficaces y baratas (Alt2)

Muy de acuerdo $(\mathrm{n}=73 ; 62,9 \%)$

Muy en desacuerdo $(\mathrm{n}=30 ; 25,9 \%)$

NS/NC ( $n=13 ; 11,2 \%)$

b) Las energías alternativas (solar, eólica, biomasa) no pueden suministrar toda la energía que se necesita en un país (Alt 3)

Muy de acuerdo $(\mathrm{n}=39 ; 33,6 \%)$

Muy en desacuerdo $(\mathrm{n}=48 ; 41,4 \%)$

NS/NC (n=29; 25,0\%)

c) La energía nuclear es la energía es segura, competitiva, limpia y garantiza el suministro eléctrico ( $\mathrm{Nucl} 1$ )

Muy de acuerdo ( $\mathrm{n}=17 ; 14,7 \%)$

Muy en desacuerdo $(\mathrm{n}=88 ; 75,9 \%)$

NS/NC ( $n=11 ; 9,5 \%)$

d) La energía nuclear debe desaparecer porque genera residuos altamente radiactivos, muy peligrosos para la salud y el medio ambiente (Nucl 2) 
Muy de acuerdo (n=81; 69,8\%)

Muy en desacuerdo $(\mathrm{n}=22 ; 19,0 \%)$

NS/NC ( $\mathrm{n}=13 ; 11,2 \%)$

e) El consumo de electricidad no afecta a la contaminación (Consumo 1)

Muy de acuerdo $(n=12 ; 10,3 \%)$

Muy en desacuerdo $(\mathrm{n}=87 ; 75,0 \%)$

NS/NC (n=17; 14,7\%)

f) Consumir menos electricidad, gas o gasolina reduciría nuestro nivel de vida (Consumo 2)

Muy de acuerdo $(\mathrm{n}=28 ; 24,1 \%)$

Muy en desacuerdo $(\mathrm{n}=75 ; 64,7 \%)$

NS/NC (n=13;11,2\%)

g) Nuestra calidad de vida mejoraría si gastáramos menos electricidad, gas o gasolina (Calidad)

Muy de acuerdo $(n=65 ; 56,0 \%)$

Muy en desacuerdo $(\mathrm{n}=34 ; 29,3 \%)$

NS/NC (n=17; 14,7\%)

h) ¿Estaría dispuesto a modificar sus hábitos de consumo y/o estilo de vida para adaptarse al proceso de cambio climático y de calentamiento global?

Sí, con toda seguridad $(n=39 ; 33,6 \%)$

Probablemente sí $(\mathrm{n}=66 ; 56,9 \%)$

Probablemente no $(\mathrm{n}=8 ; 6,9 \%)$

No, con toda seguridad ( $\mathrm{n}=1 ; 0,9 \%)$

N.S. $(\mathrm{n}=2 ; 1,7 \%)$

i) ¿Ha visto, oído o leído en algún medio de comunicación algo sobre la conveniencia o no del uso de la energía nuclear?

- Sí, en prensa $(n=10 ; 8,6 \%)$

- Sí, en tv (n=35;30,2\%)

- Sí, en Internet $(n=4 ; 3,4 \%)$

- Sí, en radio $(n=8 ; 6,9 \%)$

- Sí, en varios medios, incluida la tv ( $n=38 ; 32,8 \%)$

- No, en ningún medio ( $\mathrm{n}=21 ; 18,1 \%)$

j) ¿Se considera suficientemente informado de lo sucedido en Japón?

- Sí (n=73; 62,9\%)

- No (n=39; 33,6\%)

- No sé lo que ha pasado $(n=4 ; 3,4 \%)$

k) En caso afirmativo, tras lo sucedido ¿ha cambiado su forma de pensar al respecto del uso de la energía nuclear?

- Sí (n=62;53,4\%)

- No $(\mathrm{n}=31 ; 26,7 \%)$

- NS/NC ( $\mathrm{n}=23 ; 19,8 \%)$

1) ¿Cuántos años hace de la catástrofe de Chernóbil?

- Correcto $(\mathrm{n}=48 ; 41,4 \%)$

- Incorrecto $(n=20 ; 17,2 \%)$

- NTC/Nir $\ln =18 \cdot 111 \%$ 